\title{
Dank, wem Dank gebührt
}

Johannes Rux*

Zwei Jahre RECHTSWISSENSCHAFT. Der Abschluss des zweiten Jahrgangs ist die Gelegenheit, denjenigen zu danken, die durch ihr Engagement das Erscheinen der Zeitschrift ermöglichen. Neben den Autorinnen und Autoren, den Herausgeberinnen und Herausgebern sowie der Herstellungsabteilung des Verlags sind das vor allem die zahlreichen Gutachterinnen und Gutachter, die durch ihre Stellungnahmen entscheidend zur Qualität der Texte beigetragen haben. Das Review-Verfahren selbst ist anonym, aber diejenigen Personen, die im Jahr 2011 an der Peer Review beteiligt waren, sollen - wie schon im Vorjahr - namentlich benannt werden:

Susanne Baer (Berlin/Karlsruhe), Kilian Bälz (Kairo), Eberhard Eichenhofer (Jena), Horst Eidenmüller (München), Christoph Engel (Bonn), Andreas Fischer-Lescano (Bremen), Nicolas Forgo (Hannover), Martin Gebauer (Tübingen), Klaus Kreß (Köln), Karl-Heinz Ladeur (Hamburg), Peter Mankowski (Hamburg), Franz Merli (Graz), Lothar Michael (Düsseldorf), Magdalena Pöschl (Graz), Michael Potacs (Wien), Joachim Renzikowski (Halle), Gisela Rühl (Jena), Frank Saliger (Hamburg), Christoph Schönberger (Konstanz), Götz Schulze (Potsdam), Carl-Friedrich Stuckenberg (Saarbrücken), Jürgen Taeger (Oldenburg), Christoph Vedder (Augsburg), Andreas von Arnauld (Hamburg), Marc-Philippe Weller (Freiburg).

Uns freut es außerordentlich, dass die RECHTSWISSENSCHAFT mittlerweile sowohl in deutschen als auch in vielen ausländischen Bibliotheken geführt wird. Ihre Reaktionen bestärken uns im Bemühen, ein Forum für den fachgebietsübergreifenden Diskurs zu schaffen - und wir hoffen, dass viele Leserinnen und Leser sich zu eigenen Beiträgen angeregt fühlen. Denn neue Manuskripte sind uns natürlich höchst willkommen.

Die Kontaktdaten der Schriftleitung finden Sie unten auf Seite 2 des Heftes. Hinweise für die Manuskriptgestaltung sind auf der Internet-Seite www.rechtswissenschaft.nomos.de abrufbar.

* PD Dr. Johannes Rux lehrt Öffentliches Recht an der Eberhard-Karls-Universität Tübingen und ist Schriftleiter der RECHTSWISSENSCHAFT. 